SCIENTIFIC PUBLICATIONS OF THE STATE UNIVERSITY OF NOVI PAZAR

SER. A: Appl. MAth. Inform. AND Mech. vol. 11, 1 (2019), 41-46.

\title{
A Penteract Partition by means of the Optimal Subdivision of Cells
}

\author{
Miroslav S. Petrov, Todor D. Todorov
}

\begin{abstract}
Freudental's algorithm obtained way back in early forties have been traditionally used for simplicial triangulating of the hypercube. The main advantage of this algorithm is that it only generates one congruence class. Unfortunately, Freudental's algorithm is not optimal with respect to the measure of degeneracy. The multigrid methods require the degeneracy measure to be as small as possible. The minimal subdivision in the 3-dimensional case and the uniform tesseract corner subdivision in the 4-dimensional case are optimal in regards the measure of degeneracy and multigrid applications. The question about the optimal refinement strategy in more dimensional cases is still an open problem. This paper deals with a penteract subdivision with degeneracy measure much better than one obtained by the Freudental algorithm.
\end{abstract}

Keywords: Measure of degeneracy, congruence classes, refinement strategies.

\section{Introduction}

The rapid development of the multidimensional geometry begun after the book of Forsyth [4] had been published. The multidimensional Euclidean geometry have been widely used in various engineering applications [5]. Typical examples are some problems in the spacetime continuum and the theory of subatomic particles [7]. The finite element multigrid method for solving multidimensional boundary and eigenvalue problems needs stable subdivisions of simply connected bounded domains in $\mathbf{R}^{n}$. The subdivision of the $n$-dimensional cube plays an important role in dividing canonical domains $[8,9]$. Freudental's subdivision of the cube in combination with the red refinement strategy is widely used by researchers devoted to multigrid methods. Many authors [2] have used combinatorial approaches to obtain stable subdivisions of the cube. Todorov [9] and Petrov and Todorov [8] have proved

Manuscript received April 20, 2018; accepted December 15, 2018.

Miroslav S. Petrov and Todor D. Todorov are with the Technical University, 5300 Gabrovo, Bulgaria. 
that the Freudental partition of the cube is not optimal with respect to the measure of degeneracy in the 3-dimensional and 4-dimensional cases. Moreover, the optimal refinement strategies in these cases can not be obtained by combinatorial approaches.

In this paper we show that the same situation is in the 5-dimensional case. We divide the unit penteract into a set of identical tesseract base hyperpyramids. Each hyperpyramid is partitioned into 24 hexatera by means of optimal triangulation of the penteract boundary. Thus we obtain a new division $\mathscr{L}$ of the penteract called the cell centered partition. The division operator $\mathscr{L}$ generates two and only two congruence classes with a degeneracy measure much better than the one obtained by the Freudental algorithm [3].

\section{The cell centered penteract division}

The denotation $\left[a_{1}, a_{2}, \ldots, a_{n}\right]$ stands for a convex polytope with vertices $a_{i} i=1,2, \ldots, n$. We define the unit tesseract $T_{1}=\left\{x \mid 0 \leq x_{i} \leq 1, i=1,2,3,4, x_{5}=0\right\}$ with vertices:

$$
a_{1}(0,0,0,0,0), a_{2}(1,0,0,0,0), a_{3}(0,1,0,0,0), a_{4}(0,0,1,0,0)
$$

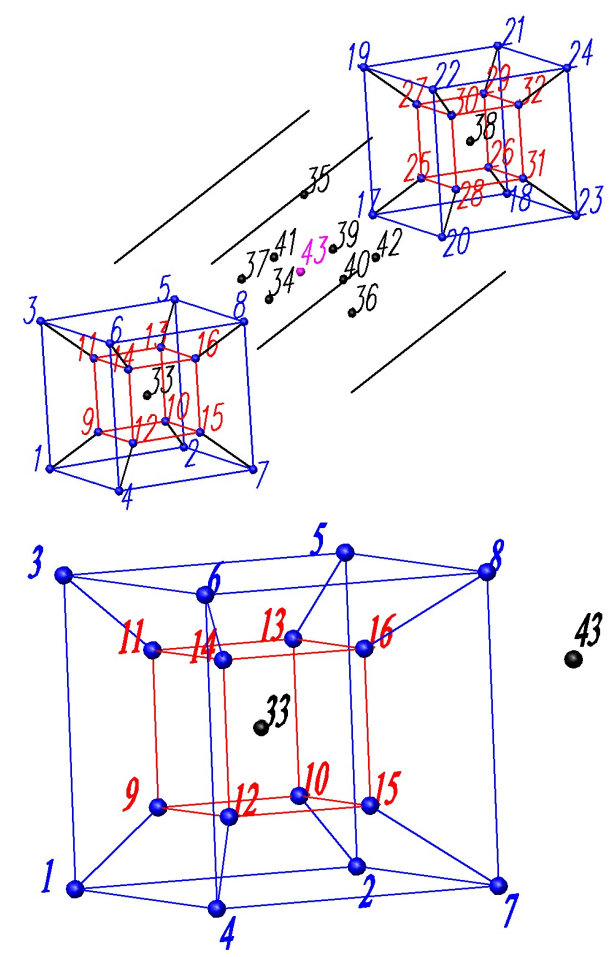

Fig. 1. The $5 D$ cube $P$ left and the cell $T_{1}$ right. 

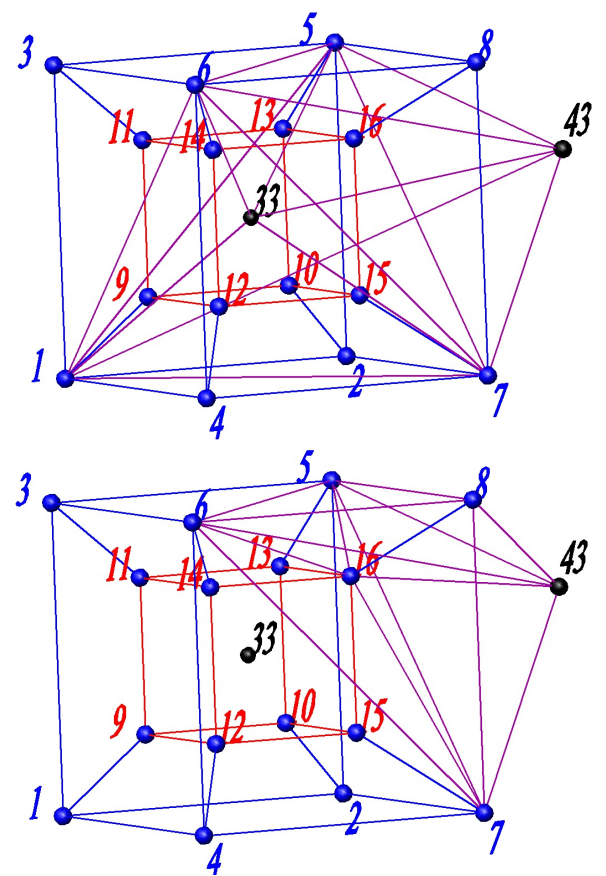

Fig. 2. A hexateron obtained by an internal tesseract corner left and another one built on external pentatope right.

$$
\begin{gathered}
a_{5}(1,1,0,0,0), a_{6}(0,1,1,0,0), a_{7}(1,0,1,0,0), a_{8}(1,1,1,0,0), \\
a_{9}(0,0,0,1,0), a_{10}(1,0,0,1,0), a_{11}(0,1,0,1,0), a_{12}(0,0,1,1,0), \\
a_{13}(1,1,0,1,0), a_{14}(0,1,1,1,0), a_{15}(1,0,1,1,0), a_{16}(1,1,1,1,0) .
\end{gathered}
$$

The tesseract $T_{10}=\left[a_{i}=a_{i-16}+(0,0,0,0,1), i=17,18, \ldots, 32\right]$ is determined by $T_{10}=$ $T_{1}+(0,0,0,0,1)$. The penteract $P=\left[a_{i}, i=1,2, \ldots, 32\right]$, Figure 1 left is the object of investigation in this section. We denote the penteract center by $a_{43}\left(\frac{1}{2}, \frac{1}{2}, \frac{1}{2}, \frac{1}{2}, \frac{1}{2}\right)$ and the center of the cell $T_{1}$ by $a_{33}\left(\frac{1}{2}, \frac{1}{2}, \frac{1}{2}, \frac{1}{2}, 0\right)$, Figure 1 right. The centers of cells $T_{i}, i=2,3, . ., 10$ are denoted by $a_{i}, i=34, \ldots, 42$ correspondingly.

Let $\mathscr{E}$ be a partition operator defined by

$$
\mathscr{E} P=\left\{H_{i} \mid H_{i}=\left[V\left(T_{i}\right), a_{43}\right], i=1,2, \ldots, 10\right\},
$$

where $V(G)$ is the set of all vertices of the polytope $G$. The polytopes $H_{i}$ are ten identical tesseract base hyperpyramids with apex the penteract center. Following Petrov and Todorov [8] we divide the tesseract $T_{i}$ optimally with respect to the measure of degeneracy. Let $\mathscr{T}$ be the partition technique [8] subdividing $T_{i}$ uniformly into 24 tesseract corners $C_{i j}$, 
$i=1,2, \ldots, 10$ and $j=1,2, \ldots, 24$. Despite all 24 elements $C_{i j}, j=1,2, \ldots, 24$ are from the same class we distinguish two kind of pentatopes belonging to $\mathscr{T} T_{i}$ :

(i) tesseract corners that satisfy $a_{i+32} \in V\left(C_{i j}\right)$;

(ii) elements that $a_{i+32} \notin V\left(C_{i j}\right)$.

We call the first kind of tesseract corners internal elements and the second kind of pentatopes external finite elements. An internal element $C_{11}=\left[a_{1}, a_{5}, a_{6}, a_{7}, a_{33}\right]$ and an external one $C_{12}=\left[a_{5}, a_{6}, a_{7}, a_{8}, a_{16}\right]$ are presented in Figure 2 . The subdivision of the boundary is determined by $\mathscr{T} \partial P=\bigcup_{i=1}^{10} \mathscr{T} T_{i}$. The set of all internal pentatopes in $\mathscr{T} \partial P$ and the set of all external tesseract corners in $\mathscr{T} \partial P$ are denoted by $I$ and $X$. We define a division operator $\mathscr{B}$ for all hyperpyramids $H_{i}$ as follows

$$
\mathscr{B} H_{i}=\left\{K_{i j} \mid K_{i j}=\left[V\left(C_{i j}\right), a_{43}\right], j=1,2, \ldots, 24\right\} .
$$

Definition 1. The cell centered penteract subdivision operator $\mathscr{L}$ is defined by $\mathscr{L}=\mathscr{B} \circ \mathscr{E}$.

Theorem 1. The operator $\mathscr{L}$ generates two and only two classes of similarity.

Proof. We suppose that the bases of all tesseract corners $C_{i j}$ are regular pyramids. The subdivision of the penteract $P$ is executed in two steps. First, we divide $P$ into ten hyperpyramids with tesseract bases and apex $a_{43}$, and second, we refine each hyperpyramid into set of simplices. The sets

$$
\begin{aligned}
& \mathscr{I}=\left\{K=\left[V(C), a_{43}\right], C \in I\right\}, \\
& \mathscr{X}=\left\{K=\left[V(C), a_{43}\right], C \in X\right\}
\end{aligned}
$$

are extensions of the sets $I$ and $X$ in the five-dimensional space. Note that $P=\mathscr{I} \cup \mathscr{X}$ and $\mathscr{I} \cap \mathscr{X}=\emptyset$.

We aim to prove that all elements of $\mathscr{X}$ are from the same class. Let us consider an arbitrary hexateron belonging to $\mathscr{X}$. For definiteness let it be $K_{12}=\left[a_{5}, a_{6}, a_{7}, a_{8}, a_{16}, a_{43}\right]$, Figure 2, right. The base $C_{12}$ is a tesseract corner with base edges equal to $\sqrt{2}$ and lateral edges equal to 1 . On the other hand all lateral edges of hexateron $K_{12}$ are equal to $\frac{\sqrt{5}}{2}$. This construction rule is valid for all elements of $\mathscr{X}$. That is why all elements of $\mathscr{X}$ are geometrically similar.

It remains to prove that the elements of $\mathscr{I}$ belong to one congruence class. We consider an arbitrary hexateron belonging to $\mathscr{I}$, for instance $K_{11}=\left[a_{1}, a_{5}, a_{6}, a_{7}, a_{33}, a_{43}\right]$, Figure 2, left. The base of $K_{11}$ is the tesseract corner $C_{11}$ with apex $a_{33}$. The lengths of all base edges of $C_{11}$ are equal to $\sqrt{2}$ and the lengths of all lateral edges are 1. There is a lateral edge of $K_{11}$ with a length $\frac{1}{2}$, which is perpendicular to the base $C_{11}$. The lengths of all other lateral edges are equal to $\frac{\sqrt{5}}{2}$. Thus can be constructed all elements of $\mathscr{I}$. Therefore they are from the same class.

Property 1. All elements of $\mathscr{P}$ have the same volume. 


\begin{tabular}{||c|c|c|c|}
\hline \hline Division operator & $\delta$ & $v$ & $p$ \\
\hline \hline $\mathscr{F} P$ & 8.56062 & 1 & 120 \\
\hline $\mathscr{L} P$ & 7.41421 & 2 & 240 \\
\hline \hline
\end{tabular}

Table 1. A comparison between different division operators.

Proof. The distance from the penteract center to each cell is the same. Moreover, all elements of $\mathscr{T} \partial P$ are from the same class and have the same volume [8]. It remains only to apply the formula for volume of a hexateron [6]

$$
\operatorname{vol} K=\frac{h \cdot \operatorname{vol} B}{5}
$$

where $K \in \mathscr{L} P, B \in \mathscr{T} \partial P$ and $h$ is the height of $K$. $\square$

We denote the measure of degeneracy of a set of finite elements $Z$ by $\delta(Z)$, the number of congruence classes by $v(Z)$ and the cardinality of $Z$ by $p$. By direct straight line computations we obtain the results in Table 1 , where $\mathscr{F}$ is the hypercube subdivision technique introduced by Freudental [3]. The cell centered partition creates only two congruence classes with measure of degeneracy $\delta(\mathscr{I})=6.24264, \operatorname{Card} \mathscr{I}=160$ and $\delta(\mathscr{X})=7.41421$, $\operatorname{Card} \mathscr{X}=80$.

\section{Conclusion}

A new two level partition of the five-dimensional hypercube is obtained. The cell centered partition method $\mathscr{L}$ has the following advantages:

(i) $\mathscr{L}$ generates a uniform triangulation of the boundary with the optimal measure of degeneracy;

(ii) the division technique $\mathscr{L}$ is superior than the Freudental's partition technique regarding the degeneracy measure $\delta(\mathscr{L} P)<\delta(\mathscr{F} P)$;

(iii) all elements of $\mathscr{L} P$ have the same volume;

(iv) it is enough to triangulate just one of the tenth hyperpyramids $H_{i}$ since the triangulations of the others can be obtained as images of affine transformations.

\section{References}

[1] R. Abedi, B. Petracovici, R. B. Haber, A space-time discontinuous Galerkin method for linearized elastodynamics with element-wise momentum balance, Comput. Methods Appl. Mech. Engrg. 195 (2006) 3247-3273.

[2] F. Ardila, C. Ceballos,Acyclic Systems of Permutations and Fine Mixed Subdivisions of Simplices, Discrete Comput Geom, 49 (2013) 485-510. 
[3] J. BEY, Simplicial grid refinement, On Freudenthal's algorithm and the optimal number of congruence classes, Numer. Math., 85(1) (1998) 1-29.

[4] A. R. Forsyth, Geometry of Four Dimensions, Cambridge University Press; First Edition edition, UK 1930.

[5] A. Inselberg, Parallel Coordinates, Visual Multidimensional Geometry and Its Applications, Springer-Verlag New York 2009, pp. 554.

[6] M. G. Kendall, A Course in the Geometry of N Dimensions, Dover Courier, 2004, 80 pages.

[7] T. MA, S. WANG, Spectral Theory of Differential Operators and Energy Levels of Subatomic Particles, J. Math. Study, 49 (3) (2016) 259-292.

[8] M. S. Petrov, T. D. Todorov, Stable Subdivision of 4D Polytopes, Numerical Algorithms, DOI: $10.1007 / \mathrm{s} 11075-017-0454-2$.

[9] T. D. Todorov, The optimal mesh refinement strategies for 3-D simplicial meshes, Computers \& Mathematics with Applications, 66 (7) (2013) 1272-1283. 